IOS Press

\title{
Editorial
}

\section{Intelligent interaction, reasoning, and applications}

\author{
Chao-Lin Liu ${ }^{a}$, Mitsunori Matsushita ${ }^{\mathrm{b}}$, Yasufumi Takama ${ }^{\mathrm{c}}$, \\ Min-Yuh Day ${ }^{\mathrm{d}}$ and Vincent Shin-Mu Tseng ${ }^{\mathrm{e}}$ \\ ${ }^{a}$ Department of Computer Science \& Graduate Institute of Linguistics, National Chengchi University, Taiwan \\ E-mail: chaolin@nccu.edu.tw \\ ${ }^{\mathrm{b}}$ Faculty of Informatics, Kansai University, Japan \\ E-mail:mat@res.kutc.kansai-u.ac.jp \\ ${ }^{\mathrm{c}}$ Faculty of System Design, Tokyo Metropolitan University, Japan \\ E-mail: ytakama@tmu.ac.jp \\ ${ }^{\mathrm{d}}$ Department of Information Management, Tamkang University, Taiwan \\ E-mail:myday@mail.tku.edu.tw \\ e Department of Computer Science and Information Engineering, National Cheng Kung University, Taiwan \\ E-mail: tsengsm@mail.ncku.edu.tw
}

Papers published in this special issue on "Intelligent Interaction, Reasoning, and Applications" of Web Intelligence were selected from the 2013 Conference on Technologies and Applications of Artificial Intelligence (TAAI 2013) and the 2013 IEEE International Workshop on Empirical Methods for Recognizing Inference in TExt (EM-RITE 2013).

Hasebe, Kawakami, Hiraoka, and Naito's work on "Card-Type Tool to Support Divergent Thinking for Embodying Benefits of Inconvenience" offers an intriguing viewpoint on system design. Tools are designed to help designers, who adopt the brainstorming approach, make originally convenient systems less convenient. Users of such less convenient systems are expected to gain other advantages in return.

Gargett and Barnden's “Gen-Meta: Generating Metaphors by Combining AI and Corpus-Based Modeling" reports an integration of multiple systems for the creation of metaphors. Metaphors for two domains, expressions of illness and political conflicts, were explored in the current system, and those exhibiting structures similar to the original expressions are ex- tracted from the corpus based on some linguistic considerations.

Li, Huang, and Shan's "Automatic Generation of Visual Story for Fairy Tales with Digital Narrative" showcases a recreational application of text analysis, Web-based search, and picture generation. Keywords are selected from statements in the stories, relevant pictures are searched from the repositories on the Internet based on their tags, and finally the pictures are composed for showing the main ideas of the original statements.

Lin, Lee, Shih, and Hsu's "Rank Correlation Analysis of RITE Datasets and Evaluation Metrics - An Observation on NTCIR-10 RITE Chinese Subtasks" analyzes the statistical relationships between the datasets used in the evaluation and the performances of the participation teams. It is well recognized that types of statement pairs influence the quality of textual entailment judgments achieved by software, and this work provides realistic clues.

We are indebted to many for the completion of this special issue. Professor Jiming Liu (the Editorin-Chief) and Professor Yuefeng Li (the Managing 
Editor) were tremendously patient for the delayed review and revision processes. The anonymous reviewers offered helpful comments for improving the manuscripts. The authors' contributions, whether accepted or not, are certainly a crucial first step for the realization of this special issue. 